\title{
Transforming the Library - Bibliotheksbau für die Zukunft
}

Published in: Vittorio Klostermann, Zeitschrift für Bibliothekswesen und Bibliographie, 60.2013, 3-4(112-122). DOI: 10.3196/18642950131253423

Der Wandel in Bibliotheken betrifft verschiedene Aspekte der täglichen Arbeit: neue Kompetenzen der Bibliotheksbeschäftigten, den Ausbau digitaler Services in einer mobilen Gesellschaft, veränderte Raum- und Bibliothekskonzepte sowie unterschiedliche Realisierungsstrategien bei der notwendigen Anpassung von vorhandenen Bibliotheken und Bibliotheksgebäuden. Der Beitrag beschäftigt sich mit aktuellen Prognosen aus den USA für zukünftige Aufgabenfelder. Wichtige Bauprojekte aus dem Ausland wurden exemplarisch ausgewählt, um aktuelle Tendenzen im Bibliotheksbau darzustellen. Die Kenntnis dieser Beispiele unterstützt vielleicht die Bibliotheksplaner und Bibliotheksplanerinnen im Alltag bei der Diskussion um die Ausgestaltung konkreter Projekte in Deutschland.

The changes taking place in libraries have an impact on various aspects of the day-today operations: new competencies of library employees, the expansion of digital services in an increasingly mobile society, changes in the physical and organizational structures, as well as different strategies for necessary adaptations of existing library operations and buildings. This article deals with the latest prognoses being discussed in the USA regarding future areas of activity. Major building projects in other countries have been chosen as being representative of the current trends in library building design. Awareness of these exemplary projects may provide support to library planners in everyday discussions on conceptual planning of specific projects in Germany. 


\section{Transforming the Library - Bibliotheksbau für die Zukunft}

Jede personelle und räumliche Veränderung in der Bibliothek, die finanzielle Auswirkungen hat, verlangt heutzutage eine stichhaltige Begründung für die Umsetzung der Maßnahme. Dabei prägt der technologische Wandel radikal die Zukunft, die seriös nur noch über kurze Zeiträume verlässlich geplant werden kann. Der Beitrag greift wichtige Entwicklungen anhand von Bauprojekten aus dem Ausland auf und weist auf zum Teil provokante Diskussionsbeiträge hin.

\section{Prognosen für die Zukunft (USA)}

Da viele Entwicklungen aus den Vereinigten Staaten mit einigen Jahren Verzögerung auch bei uns zu verzeichnen sind, macht der Blick über die Ozean Sinn. Gebäudeplanungen basieren immer auf Bibliothekskonzepten, die abhängig sind von der Ausstattung vor Ort sowie den Benutzeranforderungen an die Services der Bibliothek. Mobile Endgeräte, zukünftige Studentengenerationen und die Digitale Bibliothek verändern fast überall die Arbeit der Bibliotheksbeschäftigten, so dass eine Neuorientierung des vorhandenen Personals notwendig wird. Ein Bericht ${ }^{1}$ der Bibliothekare der University of California zum Thema „NextGen Library Staffer" beschreibt mögliche Aufgabenfelder der Zukunft:

- Collections

- Reference/Instruction

- Technical Services

- Professional Governance/Organizational Culture

Dabei wird darauf verwiesen, dass die Kenntnisse der "Technical Services" auch die Arbeit in den anderen drei Themenfeldern prägen. Nachdrücklich wird bei den detailliert aufgelisteten Skills von den Bibliothekaren gefordert, dass in "California Libraries, next-generation UC librarians must be prepared to undertake active roles in many areas of library work, while developing new skills in their primary tasks."

Auf der Charleston Conference 2011 (USA) nahm Brad Eden in seinem Vortrag "The Status Quo Has Got To Go!"3 Bezug auf den Report "Redefining the Academic Library : Managing the Migration to Digital Information Services", der zur Information der Kanzler von USamerikanischen Universitäten veröffentlicht wurde und sich mit allen Themenfeldern des Wandels in Bibliotheken beschäftigt:

- Transformational Change in the Information Landscape

- Managing the Migration to Digital Information Services

- Leveraging Digital Collections

- Rethinking the Scholarly Publishing Model

- Repurposing Library Space

- Redeploying Library Staff

\footnotetext{
${ }^{1}$ LAUC Committee on Professional Governance: The NextGen LAUC Member : A Report. 2011. Librarian Association of the University of California (LAUC)

URL: http://www.ucop.edu/lauc/committees/pg/cpg-report-2011.pdf [Stand 29.11.2012]

${ }^{2}$ Vgl. The NextGen LAUC Member : A Report. 2011. S.3 [Stand 29.11.2012]

${ }^{3}$ Blog von Don Hawkins über den Konferenzbeitrag von Brad Eden URL:

http://www.theconferencecircuit.com/2011/11/06/the-status-quo-has-got-to-go/ [Stand 29.11.2012]
} 
Der Report selber ist nicht kostenfrei verfügbar, aber Folien einer Präsentation ${ }^{4}$ über den Bericht.

Der Wandel in der Informationslandschaft wird mit vier Schlagworten beschrieben und mit entsprechenden Zahlen anschaulich untermauert. Die steigenden Kosten und die sinkenden Finanzmittel bringen selbst Elite-Universitäten ${ }^{5}$ in Schwierigkeiten, Öffnungszeiten und Medienangebot in gleicher Weise aufrecht zu erhalten. Dazu kommt bei einem günstigenfalls festen Erwerbungsbudget dennoch die Verdrängung der Bücher durch die Zeitschriften, da bedingt durch die Preissteigerungsraten immer mehr Budget für die Zeitschriften gebunden wird $^{6}$. Die Bemühungen der Bibliotheken, ihre gedruckten Bestände zu digitalisieren, wird verglichen mit Google Books, ein finanziell und technisch extrem leistungsfähiges Programm, das bereits riesige Mengen digitalisierter Bestände zur Verfügung stellt und damit langfristig eine (kostenpflichtige?) Plattform für Informationssuchende darstellt: „Our ultimate goal is to work with publishers and libraries to create a comprehensive, searchable, virtual card catalog of all books in all languages that helps users discover new books and publishers discover new readers." 7 Schon heute nutzen Studierende als Sucheinstieg vorrangig Google, aber auch die Wissenschaftlerinnen und Wissenschaftler nutzen den klassischen Bibliothekskatalog erst nachrangig. ${ }^{8}$

Die Bestandsgröße (vor allem des gedruckten Bestandes) wird als Kriterium zur Qualitätsmessung einer Bibliothek zunehmend unwichtiger werden, denn urheberrechtsfreies Material wird digital verfügbar sein, just-in-time Zugriff auf kostenpflichtige elektronische Informationsquellen an Bedeutung gewinnen.

Universitäten auch in Deutschland werden am Studienerfolg der Studierenden oder am Einwerben von Forschungsmitteln gemessen - was trägt die Bibliothek dazu bei, hier erfolgreich zu sein? Dafür sind neue Qualitätskriterien zu entwickeln. Dazu ist in den traditionellen Aufgabenfeldern eine Abnahme der Präsenznutzungen in der Bibliothek zu verzeichnen, z.B. bei den Ausleihen oder bei den Anfragen zu den Nachschlagewerken und Bibliographien. Dafür gibt es heute neue Anforderungen seitens der Studierenden und der Wissenschaft: die Bibliotheken werden zunehmend als Ort des Lernens und der sozialen Kontakte wahrgenommen, die Wissenschaftlerinnen und Wissenschaftler wünschen sich Unterstützung beim Publikationsprozess und bei der Förderung von Medien- und Informationskompetenz.

Bezogen auf die Entwicklung bei den Bibliotheksgebäuden wird in dem Report eine Optimierung der Nutzung der vorhandenen Flächen empfohlen, z.B. bei großen Freihandbeständen eine Reduktion zugunsten von Flächen für Studierende und Fakultätsangehörige. Die Lagerung von Büchern in Freihandmagazinen auf dem Campus ist nach Aussagen der Autoren des Reports fünf Mal teurer als eine Lagerung in einem Speichermagazin außerhalb des Campus. Bei der langfristigen Speicherung ist eine Duplizierung von gedruckten Beständen - auch über Institutionen hinweg - natürlich zu vermeiden.

Die Empfehlungen des Reports „Redefining the Academic Library : Managing the Migration to Digital Information Services” listen zusammengefasst folgende Veränderungen auf:

\footnotetext{
${ }^{4}$ Auszug Foliensatz über den Report "Redefining the Academic Library : Managing the Migration to Digital Information Services" unter URL: http://www.theconferencecircuit.com/wp-content/uploads/Provosts-Report-onAcademic-Libraries2.pdf [Stand 29.11.2012]

${ }^{5}$ UCLA Library Budget 2009-10. URL: http://blogs.library.ucla.edu/universitylibrarian/2009/08/04/ucla-librarybudget-2009-10/ [Stand 29.11.2012]

${ }^{6}$ Vgl. Foliensatz "Redefining the Academic Library : Managing the Migration to Digital Information Services", Folie Nr. 11

${ }^{7}$ URL: http://books.google.de/googlebooks/library.html [Stand 10.12.2012]

${ }^{8} \mathrm{Vgl}$. Foliensatz "Redefining the Academic Library : Managing the Migration to Digital Information Services",, Folie Nr. 17
} 
- Reduktion des Angebots von gedruckten Büchern im Freihandbereich der Bibliothek

- mobiler Zugriff auf die Bibliothekbestände und die Bibliotheksservices

- die Flächen sind flexibel und modular nutzbar zu gestalten

- eine angemessene, komfortable Ausstattung der Publikumsbereiche fördert die Zusammenarbeit, insbesondere für die Studierenden

- für die Wissenschaft sollte ein neues Raumangebot innerhalb des vorhandenen Gebäudes für neue Services geschaffen werden sowie für die Allgemeinheit Aufenthaltsbereiche wie z.B. Cafés mit Angeboten zum Essen und Trinken

Vielleicht werden vor diesem Hintergrund die alten Empfehlungen des Wissenschaftsrates zum Magazinbedarf wissenschaftlicher Bibliotheken ${ }^{9}$ (1986) aufgrund der fehlenden Finanzmittel auch in Deutschland noch Realität? Die Aussonderung von Beständen ist in Deutschland bereits kein Tabuthema mehr.

\section{Frei-Räume (Lausanne, $\mathrm{CH}$ )}

Eines der aktuell architektonisch herausragenden Bibliotheksgebäude ist das „Rolex Learning Center"10 in Lausanne. Das in der Mitte des Campus der École Polytechnique Fédérale de Lausanne EPFL gelegene Gebäude ist das Zentrum für ca. 9.500 Studierende, 2.000 PhD-Studierende, 370 Professorinnen und Professoren sowie ca. 5.000 Mitarbeiterinnen und Mitarbeiter. Es sollte nicht nur die Zusammenlegung von Teilbibliotheken ermöglichen, sondern ein Gebäude mit besonderer Aufenthaltsqualität sein. Das japanische Architekturbüro SANAA mit den Architekten Kazuyo Sejima und Ryue Nishizawa gewann 2004 den Wettbewerb, 2007 - 2009 wurde das Gebäude errichtet und im November 2009 der Bibliothek zum Bezug übergeben. Im Mai 2010 erfolgte dann die feierliche Übergabe des Gebäudes. Die Finanzierung wurde mit 100 Mio. SFr geplant, dabei übernahmen Sponsoren 50\% des Betrages!

Im Gegensatz zu den umliegenden mehrgeschossigen Gebäuden entwarf SANAA einen großen, eingeschossiges flachen Bau mit den Abmessungen 166,5 x 121,5 m, bestehend aus zwei Schalen mit 14 Einschnitten, offenen Innenhöfen und phantastischen Ausblicken auf die Berge und den See. Durch die partielle Krümmung des Gebäudes und die daraus entstehenden öffentlichen Freiräume können Fußgänger das Gebäude ohne Stufen queren und so zum Genfer See gelangen. Das Gebäude ist fast zur Gänze ein einziger großer Raum, wirklich abgetrennt ist nur das Audimax. Im Untergeschoss befinden sich die Toiletten, Technikräume sowie eine Tiefgarage mit 500 Stellplätzen.

\footnotetext{
${ }^{9}$ Vgl. Wissenschaftsrat: Empfehlungen zum Magazinbedarf wissenschaftlicher Bibliotheken. Bonn, 1986. URL: http://digital.ub.uni-paderborn.de/download/pdf/465047?name=Empfehlungen Magazinbedarf wissenschaftlicher Bibliotheken [Stand 10.12.2012]

${ }_{10}^{10}$ Vgl. Della Casa, Francesco; Perret, Jacques: Rolex Learning Center : English Guide. Lausanne: EPFL Press, 2012
} 


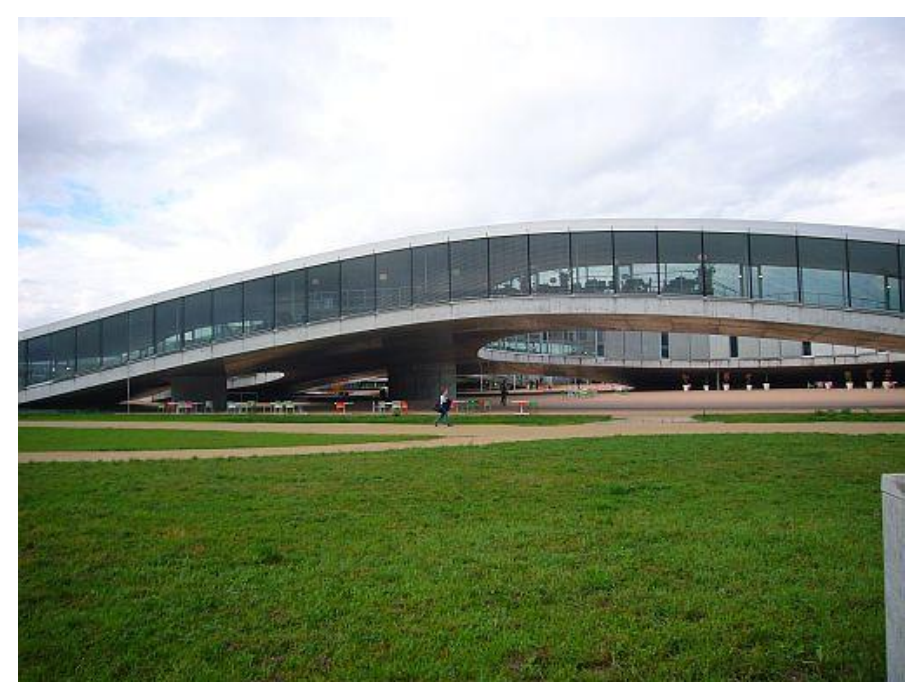

Abb. 1: Rolex Learning Center: Fassadenausschnitt, Blickrichtung von der Seeseite (Foto: Feldsien-Sudhaus)

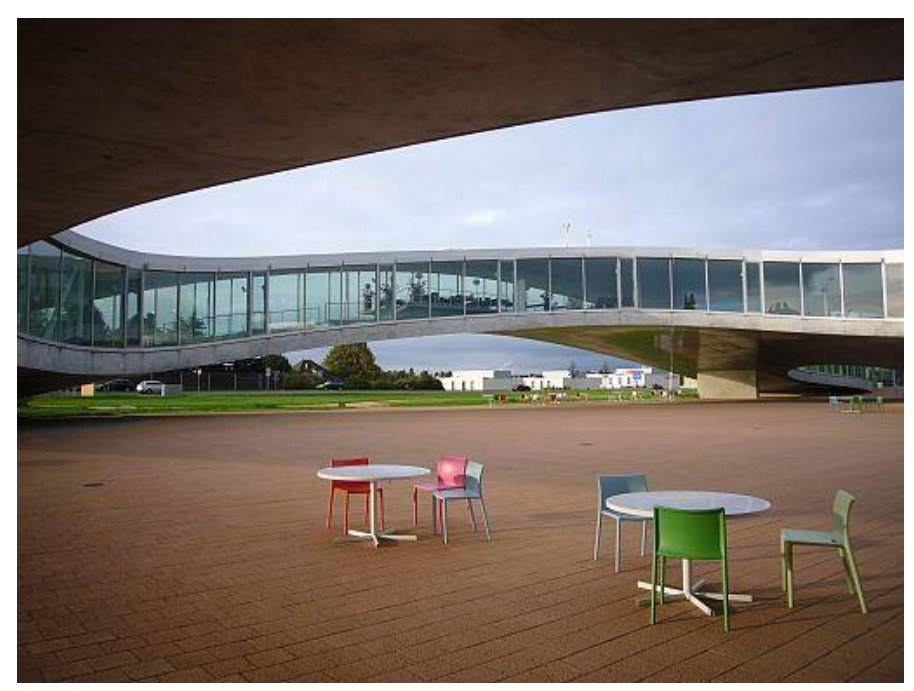

Abb. 2: Rolex Learning Center: Das Restaurant thront auf dem höchsten Punkt mit der besten Aussicht (Foto: Feldsien-Sudhaus)

Das Raumprogramm umfasst ein Auditorium „Forum Rolex“ mit 600 Plätzen, die Bibliothek mit ihren 500.000 Bänden sowie den 860 Arbeitsplätzen mit WLAN-Zugang, ein Restaurant (80 Plätze), eine Selbstbedienungs-Cafeteria (120 Plätze) sowie eine Café-Bar (80 Plätze), dazu ein Buchladen, ein Verlag, eine Bankfiliale, diverse Büros sowie ein Ausstellungsbereich. Das gesamte Gebäude verfügt nur über gläserne Außenwände, wobei die Gläser aufgrund des steigenden und abfallenden Fußbodens jeweils individuell gefertigt werden mussten. Notwendige Abtrennungen innerhalb des Gebäudes wurden mit Glas (z.B. Gruppenarbeitsräume), Streckmetallgitter (z.B. beim Buchladen und den Arbeitsflächen der Beschäftigten) oder mit in den Raum gestellten, geschlossenen weißen Zylindern (z.B. das Büro für die Alumni-Betreuung und die Bank) gestaltet. Der fast überall geneigte Fußboden stellte natürlich für die Barrierefreiheit sowie für den Transport von Waren und Büchern eine besondere Herausforderung dar. Dies wurde dadurch gelöst, dass Schrägaufzüge zur Überbrückung von Steigungen eingebaut wurden. Auf eingezogenen Terrassen stehen Tische und Regale, lange Rampen überbrücken die Niveauunterschiede. Die Bibliotheksflächen sind nicht überall vom allgemeinen Publikumsverkehr baulich abgetrennt, 
was der Bibliothek punktuell auch Schwierigkeiten bereitet. Es gibt viele Freiflächen, die zum zwanglosen Treffen einladen, Sitzkissen bieten Möglichkeiten zum Relaxen auf dem Fußboden bei schönen Ausblicken in die Landschaft. Das Restaurant mit Bedienung thront auf einer Terrasse vis-a-vis den Bergen. Das Gebäude wird damit dem Anspruch der Hochschule gerecht, nicht nur funktional Einrichtungen der Hochschule zu beherbergen, sondern als Treffpunkt und Aufenthaltszone für alle Mitglieder der Hochschule und auch der Öffentlichkeit zu fungieren.

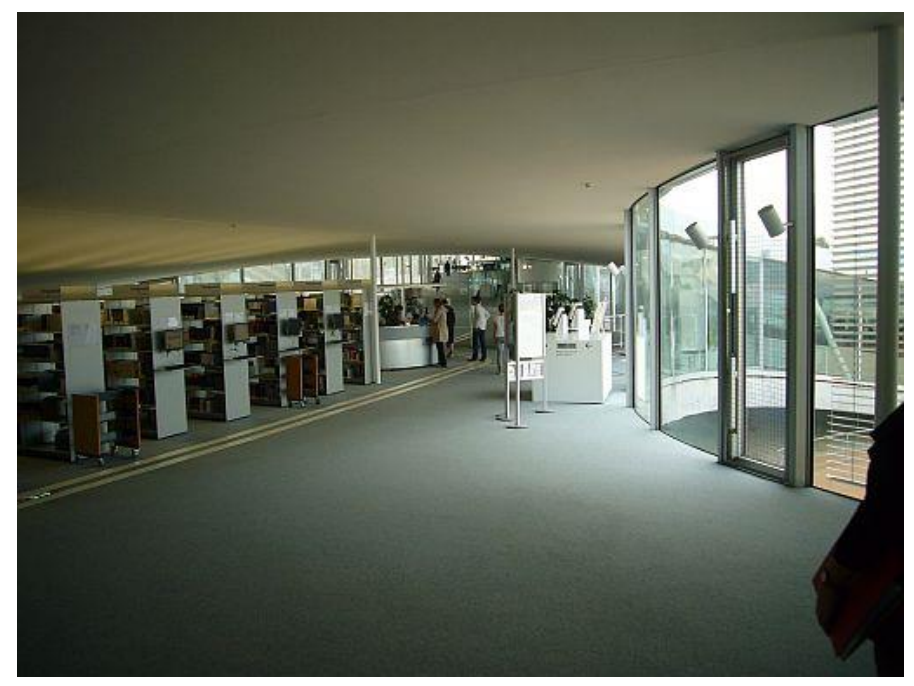

Abb. 3: Rolex Learning Center: Blick aus der Bibliothek zum Bibliothekseingang mit Information und Selbstausleihe (Foto: Feldsien-Sudhaus)

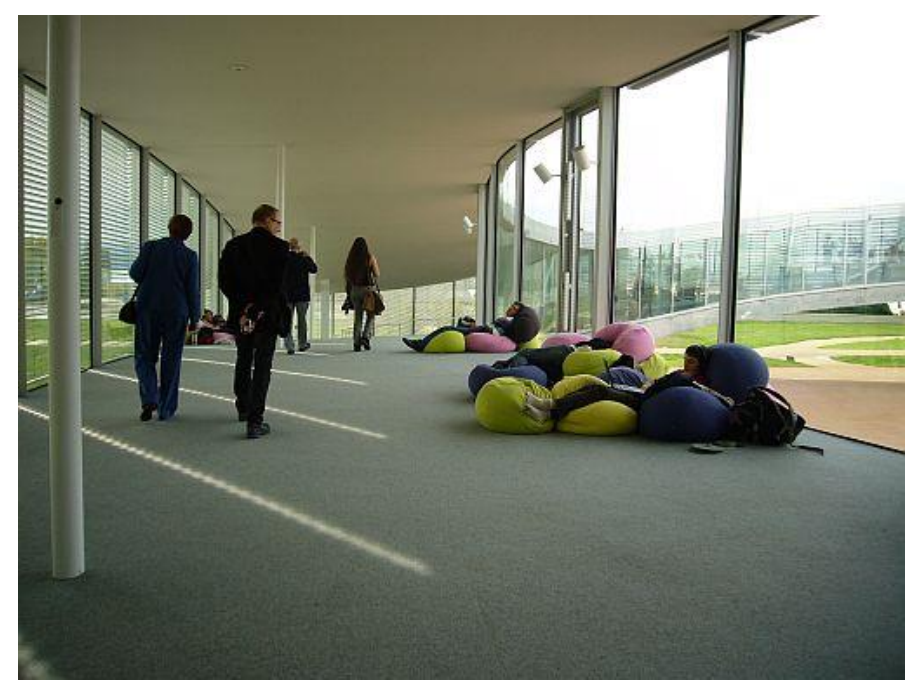

Abb. 4: Rolex Learning Center: Offener Raum zum Arbeiten oder Relaxen (Foto: Feldsien-Sudhaus) 


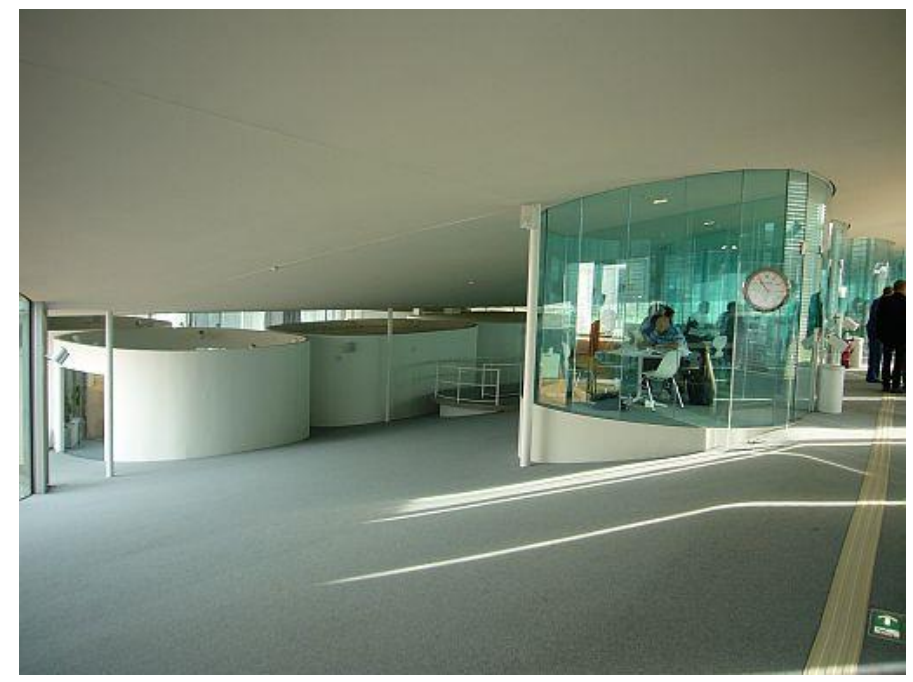

Abb. 5: Rolex Learning Center: Gläserne Gruppenarbeitsräume (Foto: Feldsien-Sudhaus)

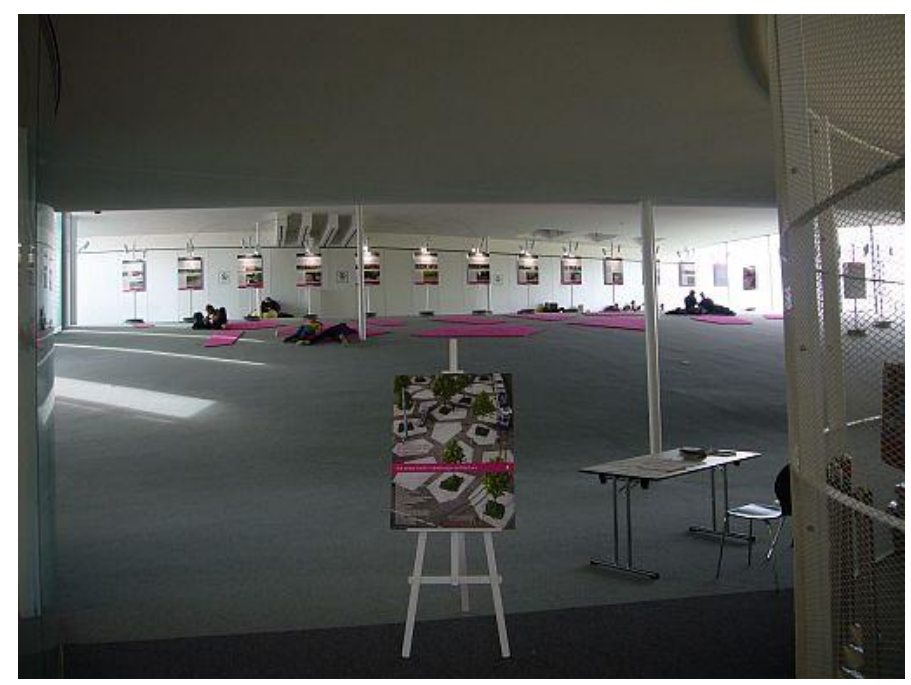

Abb. 6: Rolex Learning Center: Ausstellungszone mit Relax-Angeboten (Foto: Feldsien-Sudhaus)

\section{Schritt-für-Schritt (Clemson, USA)}

Bei vielen Nachkriegsgebäuden in Deutschland stehen jetzt umfangreiche Sanierungen an, insbesondere der (sicherheits-)technischen Infrastruktur. Dazu kommen vielfach auch energetische Sanierungen der Außenhaut und Gebäudeerweiterungen. Bei einigen Projekten wird das gesamte Gebäude während der Bauzeit geräumt. Alternativ gibt es auch Beispiele für eine bereichsweise Sanierung bei laufendem Betrieb der Bibliothek, was in der Regel den Bauprozess verlängert, aber den Vorteil bietet, dass erste Maßnahmen schnell sichtbar werden.

Ein schönes Beispiel für eine schrittweise Erneuerung ist an der Clemson University ${ }^{11}$ die Planung der Sanierung der Robert Muldrow Cooper Library ${ }^{12}$. In dem 1966 errichteten, nach

\footnotetext{
${ }^{11}$ Adresse: Clemson, South Carolina 29634, USA. URL: http://www.clemson.edu/ [Stand 03.01.2013]

${ }^{12}$ Wall, Kay L.: Clemson's Road Map - A phased design strategy aims at long term goals while enabling incremental change now. In: Library Journal Nov 15, 2011

URL: http://www.libraryjournal.com/lj/home/892659-264/clemsons_road_map.html.csp\#road [Stand 10.12.2012]
} 
damaligen Maßstäben hoch modernen Gebäude halten sich heute innerhalb von 24 Stunden bis zu 10.241 Besucherinnen und Besucher ${ }^{13}$ auf. Neben der Sanierung wurde eine Erweiterung der Bibliothek geplant und durch Architekten ein Masterplan erarbeitet. Die Kostenschätzung für das Projekt lag bei 70 Mio. Dollar. Ende Oktober 2008 wurde der Masterplan der Universitätsleitung vorgestellt, eine Woche später stoppte die Finanzkrise das Vorhaben. Die Bibliothek musste erkennen, dass das Bestehen auf der Umsetzung des Masterplans für die weitere räumliche Entwicklung der Bibliothek ein „Alles oder Nichts“ bedeutet hätte, also eine unsichere Zukunft.

Die Teilnahme an einem Bauseminar 2010 gab Impulse zur Veränderung der Strategie: Dort wurde von dem Architekturbüro Craig Gaulden Davis ${ }^{14}$ der Umbau der 1993 eröffneten Richland County Public Library ${ }^{15}$ vorgestellt, der eine gewünschte Flächenerweiterung für bestimmte Services ohne weitere bauliche Erweiterung beinhaltet. Inzwischen waren einige Annahmen des Masterplans, z.B. zur Bestandsentwicklung, überholt, so dass die Bibliothek sich entschied, gemeinsam mit dem Architekturbüro Craig Gaulden Davis eine neue Planung aufzustellen und eine RoadMap ${ }^{16}$ für Umbauten innerhalb des vorhandenen Gebäudes zu erarbeiten. Der Vorteil: Die Veränderung in Schritten lässt sich gut visualisieren, es können bereits mit geringen Mitteln erste Ergebnisse nach außen sichtbar werden. Der Nachteil liegt in der längeren Realisierungsdauer. Da gerade Universitäten in ihrer Entwicklung dynamisch sind, erfordern Planungen anhand einer Roadmap eine regelmäßige Evaluation der Ziele der Universität und ihrer Serviceeinrichtung Bibliothek, damit die Planungen nicht auseinanderlaufen. Alle Bibliotheken der University of Clemson haben zwischenzeitlich gemeinsam Leitideen für den Wandel bis zum Jahr $2020^{17}$ erarbeitet. Die eingeschlagene Strategie ist bisher erfolgreich gewesen, die Robert Muldrow Cooper Library erhielt für erste Maßnahmen in 2011-2012 insgesamt 200.000,- Dollar aus einem Sanierungsfond!

\section{Transition - aufgabenbezogen und räumlich (Kopenhagen, DK)}

Der Einzug der Digitalen Welt in Bibliotheken verändert auch die großen, nationalen Bibliotheken. Als ein Beispiel dafür soll hier die Königliche Bibliothek Kopenhagen ${ }^{18}$ vorgestellt werden, die 1648 gegründet wurde und seit 1989 auch als Universitätsbibliothek für die Versorgung der Universität von Kopenhagen zuständig ist.

Der als „Schwarzer Diamant“ bezeichnete Erweiterungsbau der Königlichen Bibliothek in Kopenhagen ist seit 1999 in Betrieb. 1993 wurde das Raumprogramm für den Wettbewerb erstellt. Damals waren die räumlichen Konsequenzen durch die Ausbreitung des Web noch nicht absehbar, aber die Bibliothek bemühte sich bereits zu dieser Zeit um große innere Flexibilität des Gebäudes. Dabei bleiben bis heute die markante Kubatur, die Außenwände und Glasfassaden stets unverändert, die Nutzung der Innenräume kann aber angepasst oder verändert werden. Das kann einfach eine Veränderung des Mobiliars beinhalten, z.B. den Abbau von Regalen des klassischen Zeitungs- und Zeitschriftenlesesaals zugunsten weiterer Nutzerarbeitsplätze mit Laptop als Studienraum für eMedien und Datenbanken. Es kann

\footnotetext{
${ }^{13}$ Stand: September 2011. siehe ${ }^{12}$

${ }^{14}$ URL: http://www.cgdarch.com/ [Stand 10.12.2012]

${ }^{15}$ Adresse: Richland Library, 1431 Assembly Street, Columbia, SC 29201. USA URL: http://www.richlandlibrary.com/locations/main [Stand 03.01.2013]

${ }_{17}^{16}$ URL: http://media.clemson.edu/library/lib_overview/plans/CooperLibraryRoadMapBook.pdf [Stand 10.12.2012]

${ }^{17}$ Clemson University Libraries: 2020 Planning Document. URL:

http://media.clemson.edu/library/lib_overview/plans/LibrariesFINAL2020documentupdatejune 2011.pdf [Stand 10.12.2012]

${ }^{18}$ Det Kongelige Bibliotek. Nationalbibliotek og Københavns Universitetsbibliotek : Hovedpunkter af mere end 500 års historie. April 2007. URL: http://www.kb.dk/export/sites/kb_dk/da/kb/historie/historie/historie2007.pdf [Stand 10.12.2012]
} 
aber auch umfangreichere Umorganisationen ${ }^{19}$ beinhalten. Der Raum für die Sondersammlung Karten und Fotos wird jetzt auch als Versammlungsraum verwendet. Das Gebäude der Bibliothek ist eine Touristenattraktion und zieht viele Besucherinnen und Besucher an. Nachdem der Buchladen im Eingangsbereich des Gebäudes im Jahr 2011 aufgab, wird diese Fläche seit Februar 2012 für die „Diamant-Information“ genutzt. Da die jährlichen Ausleihen von 250.000 auf 25.000 Ausleihen sanken, wurde auch hier die Leihstelle umgebaut und reduziert. Dabei wurde berücksichtigt, dass das großzügige Foyer mit Blick auf das Wasser alljährlich für die Erstsemester-Party zur Begrüßung der Studierenden der Kopenhagener Hochschulen genutzt wird und damit die Bibliothek zu einer begehrten Event-Location geworden ist.

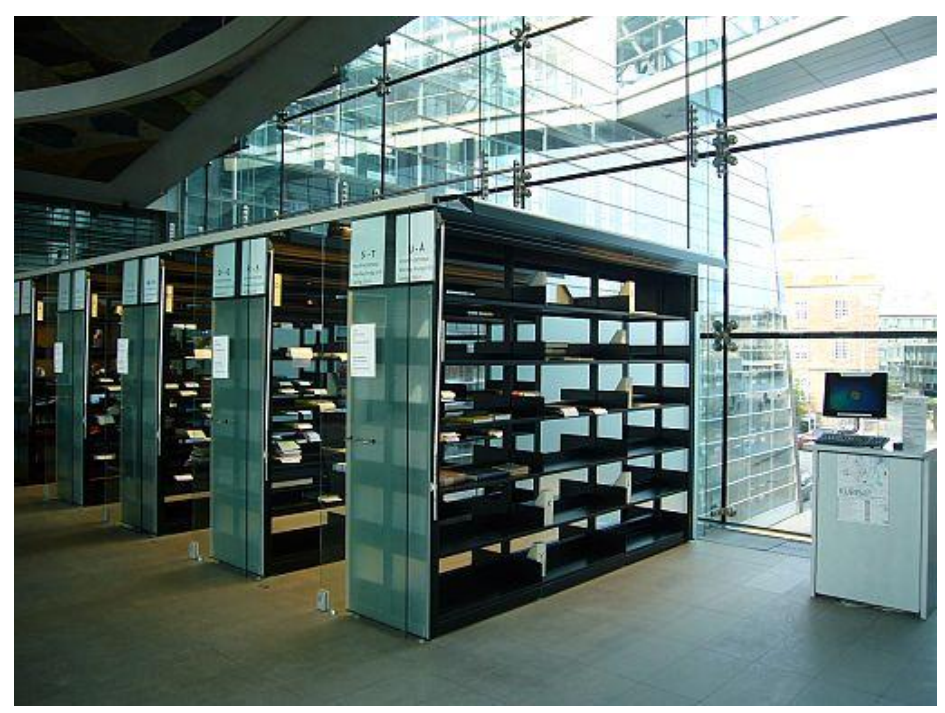

Abb. 7: Königliche Bibliothek Kopenhagen : Die zur Selbstausleihe umgebauten Ausleihregale mit verschließbaren Glasschiebetüren (Foto: Feldsien-Sudhaus)

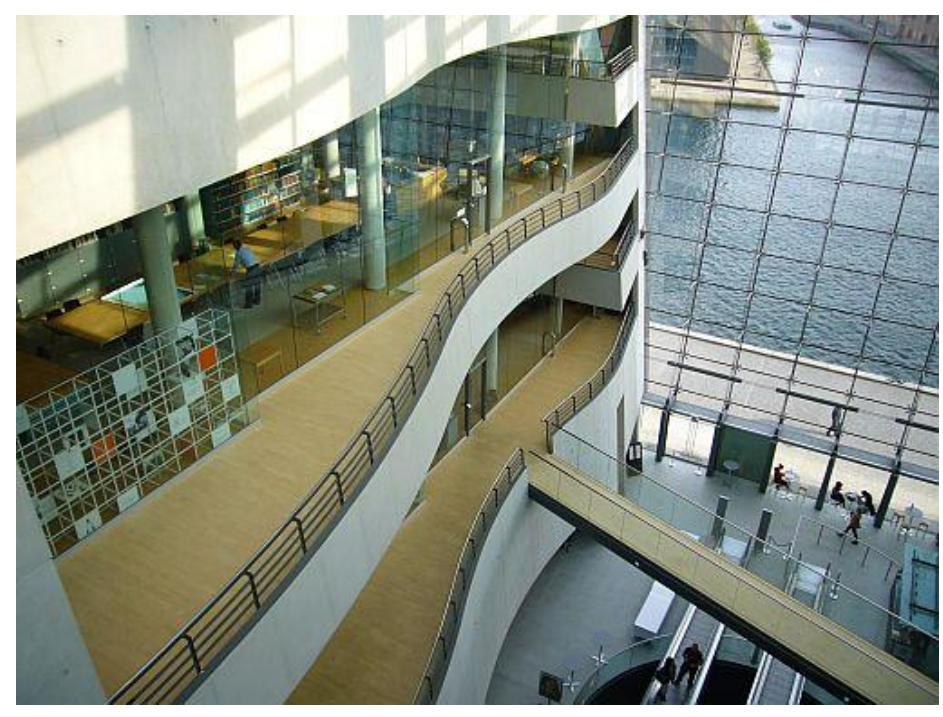

Abb. 8: Königliche Bibliothek Kopenhagen : Links der Sonderlesesaal Karten und Fotos, der heute zur Meeting Hall umgebaut ist (Foto: Feldsien-Sudhaus)

\footnotetext{
${ }^{19}$ Larsen, Steen Bille: Refurbishing The Black Diamond to meet the digital challenges. Vortrag 19.04.2012, 16. Seminar of LIBER Architecture Group LAG. URL: http://147.88.230.242/liberlag/PP LAG 12/Thursday/SBL Black Diamond Prague Liber 2012 SBLarsen vorbereitet.pdf [Stand 10.12.2012]
} 


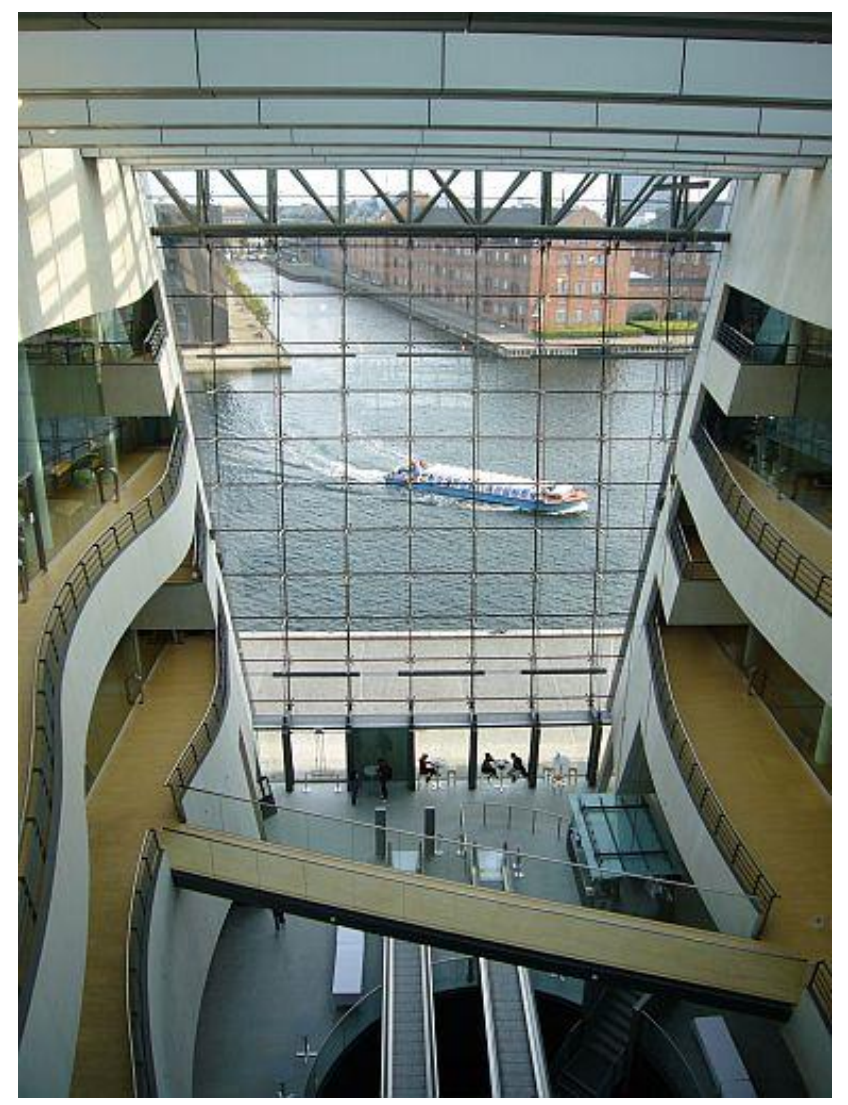

Abb. 9: Königliche Bibliothek Kopenhagen : Blick in das am Wasser gelegene zentrale Foyer, das auf allen Ebenen als Event-Location genutzt wird (Foto: Feldsien-Sudhaus)

\section{Innovate - Educate - Create $^{20}$ (Delft, NL)}

Ein sehr bekanntes Beispiel für moderne Bibliotheksarchitektur ist das Gebäude der Universitätsbibliothek der TU Delft von den Architekten Mecanoo ${ }^{21}$. Der Originalzustand von $1998^{22}$ umfasste 15.000 qm Fläche mit dem Lesesaal, einem abgeschlossenen, separat zugänglichen gläsernen Raum mit Computer-Arbeitsplätzen, einem Sonderlesesaal, einem großen unterirdischen Magazinbereich, Büros für die Bibliotheksverwaltung, dem

Universitätsverlag, der Buchbinderei und einem Buchladen. Das Gebäude wird auch heute noch markiert durch das schräge, begehbare begrünte Dach. In dieses Dach ist ein großer Kegel eingeschnitten, der auch optisch in der zentralen Halle des Lesebereichs präsent ist. Im Kegel sind auf mehreren Galerien Leseplätze angeordnet, in der Eingangsebene befand sich unterhalb des Kegels eine große Thekenanlage für die zentrale Auskunft und Ausleihe. Beim Betreten der Bibliothek dominierte daneben eine große Bücherwand mit 60.000 Bänden Freihandbestand, auf der linken Seite der Halle befand sich eine Ausstellung mit allen laufenden Print-Zeitschriftenabonnements. Technisch wurde das Gebäude als „grüne Bibliothek" konzipiert: Über Wärmetauscher wird das Grundwasser und die Erdwärme genutzt, eine Doppel-Glas-Fassade sorgt für gutes Klima in den dahinter liegenden Büros, das grüne Dach verringert die Versiegelung der Flächen.

\footnotetext{
${ }^{20}$ van Wezenbeek, Wilma: TU Delft Library - Educate. Innovate. Create. Vortrag 16.04.2010, 15. Seminar der LIBER Architecture Group LAG URL: http://web.zhbluzern.ch/liber-lag/PP_LAG_10/Friday/Wilma_v_Wezenbeek100416_bearb_un.pdf [Stand 28.12.2012]

${ }^{21}$ URL: http://www.mecanoo.nl/ [Stand 28.12.2012]

${ }^{22}$ Planung des Gebäudes von 1993-1996
} 
Als die Universität Delft mit den Planungen für ein neues Learning Center startete, wurde auch die Bibliothek bei den Planungen mit einbezogen. Die Anforderungen der Nutzerschaft und neue Services verlangten nach gut zehn Jahren Betrieb bereits wieder nach neuen Konzepten: die Idee eines Library Learning Centre wurde in Abstimmung mit den zentralen Planungen entwickelt. Die gemeinsame Arbeitsgruppe besuchte und studierte verschiedene, relevante Projekte, führte Gespräche mit Firmen, Architekten und Nutzergruppen. Da die Bibliothek auch nationale Aufgaben wahrnimmt, wurden als übergeordnete Zielgruppen der Bibliothek die Studierenden der TU Delft, die wissenschaftlichen Belange der Universität sowie die Öffentlichkeit und der Staat bei den Planungen berücksichtigt, was 2007 ausführlich dokumentiert wurde. Das Library Learning Center sollte zukünftig verbunden werden mit den Zielen:

- einen Platz zum Studieren schaffen

- das Center soll attraktiv sein und als Vorreiter fungieren

- ein Wohnzimmer für alle Besuchergruppen darstellen

- eine Schatzkammer bei der Nutzung der Sondersammlungen bieten

- ein Lotse beim Wissensmanagement sein

- Raum und Mobiliar sind in der Gestaltung und Nutzung flexibel

Mit einer Ausstellung 2008 und in einem Blog wurden die Nutzerinnen und Nutzer informiert und einbezogen. Der geplante Wande ${ }^{23}$ erforderte räumliche Veränderungen insbesondere in der zentralen Halle, aber auch in der Organisation der Services. Zunächst wurde in 2009 die große zentrale Thekenanlage verkleinert, da die Verbuchung nun über RFIDSelbstverbuchungs-Terminals erfolgt. Der Raum der Präsentation der aktuellen Zeitschriftenabonnements wurde u.a. für Computer-Arbeitsplätze umgenutzt. Im großen gläsernen Saal wurden für die geforderten Still-Arbeitsplätze zum Teil die Computer abgebaut und die mit Abschirmungen versehenen Tische gegen andere Tische zur Nutzung mit mobilen Geräten ausgetauscht. Die befragten Studierenden hatten aber auch den Wunsch geäußert, dass weiterhin Computer zum Arbeiten zur Verfügung gestellt werden sollten. Es gibt vier kleine Gruppenarbeitsräume, die individuell mit themenbezogener Einrichtung ein besonderes Ambiente schaffen. Der Zugang zu den Sondersammlungen wurde umgestaltet und ist für Besucherinnen und Besucher damit besser wahrnehmbar. Als besonderes Highlight wurde eine kleine "Cafe Corner" integriert.

Ein zweiter, großer Schritt waren die organisatorischen Veränderungen: die Benutzerinnen und Benutzer werden mehr als Gäste des Hauses angesehen, Services damit nicht mehr an einen festen Ort gebunden sondern die Bibliotheksbeschäftigten sind im Gebäude mobil und ohne Barriere ansprechbar. Eine zentrale Rolle kommt auch der umgestalteten

Auskunftstheke zu, die heute mit drei Arbeitsplätzen besetzt ist: der zentralen Auskunft sowie der Hilfe für IT-Fragen und während der Examenszeiten einem Platz für die Hilfestellung bei Fragen zur Mathematik, einem Fach, das vielen Studierenden an den Technischen Universitäten Probleme bereitet. Damit bietet die Bibliothek nicht nur Raum und Informationsquellen, sondern auch Unterstützung beim Lernprozess.

\footnotetext{
${ }^{23}$ URL: https://intranet.tudelft.nl/services/projecten/tu-delft-projecten/project-learning-centre/transformatie-librarylearning-centre/ [Stand 28.12.2012], Fotos zum Projekt sind zugänglich unter: http://www.flickr.com/photos/tudelftlibrary/page50/
} 


\section{Umnutzung des Postgebäudes (Luzern, $\mathrm{CH}$ )}

Zwischen dem modernen Bahnhofsgebäude des spanischen Architekten Calatrava und dem am Luzerner See gelegenen Kultur- und Kongresszentrum von Jean Nouvel befindet sich das 1981-1985 erbaute ehemalige Postbetriebsgebäude der Architekten Ammann und Baumann, Luzern. Als dieses in so prominenter Lage gelegene Gebäude von der Post aufgegeben werden sollte, war das die Chance für die Universität Luzern und die Pädagogische Hochschule Luzern (PHZ), ihre Platzprobleme zu lösen. Sie verabschiedeten sich damit von älteren Planungen, die nie realisiert werden konnten. Die Zentral- und Hochschulbibliothek Luzern (ZHB $)^{24}$ ist für die bibliothekarische Versorgung beider Einrichtungen zuständig und wurde daher auch in die Planungen einbezogen. Der Standort ermöglichte in einem Gebäude die Versorgung aller 2.600 Studierenden der Universität mit ihren drei Fakultäten - Kultur- und Sozialwissenschaften, Rechtswissenschaften und Theologie - sowie Teile der Pädagogischen Hochschule mit 1.000 Studierenden. Das Raumprogramm umfasste die Büros der Fakultäten, eine Mensa, ein Audimax sowie Hörsäle. In dem 2005 durchgeführten Wettbewerbsverfahren gewann das Büro Enzmann + Fischer AG, Zürich, das die Baumaßnahme betreute. Es gelang dabei, die bisherigen 24 Standorte der Universität in einem Gebäude zu vereinigen. Das beinhaltete die Zusammenlegung der verschiedenen, über die Stadt verteilten Bibliotheksstandorte.

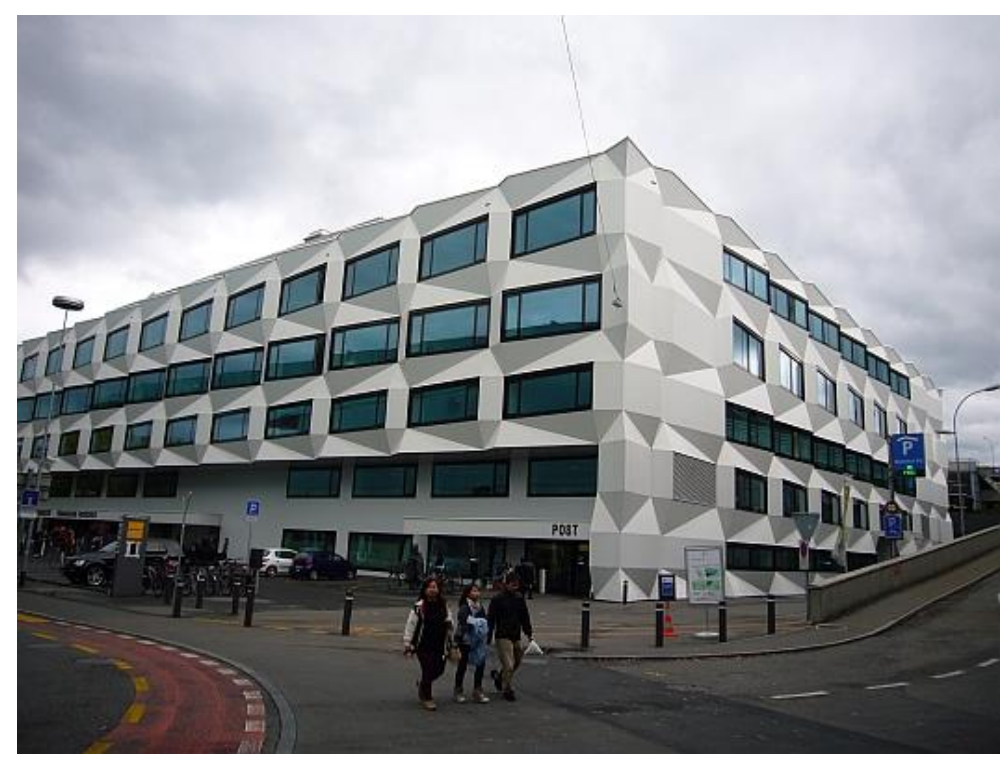

Abb. 10: Uni/PH Luzern : Die neue Fassade des ehemaligen Postgebäudes (Foto: Feldsien-Sudhaus)

\footnotetext{
${ }^{24}$ ZHB im UNI/PHZ-Gebäude. Der neue ZHB-Standort - die grosse wissenschaftliche Bibliothek im Zentrum Luzerns. findemittel UNI/PHZ-Gebäude. URL: http://www.zhbluzern.ch/fileadmin/zhbluzern/ueber_uns/bauen_zuegeln/dok/Findemittel-UPG_2011-1.pdf [Stand 29.12.2012]
} 


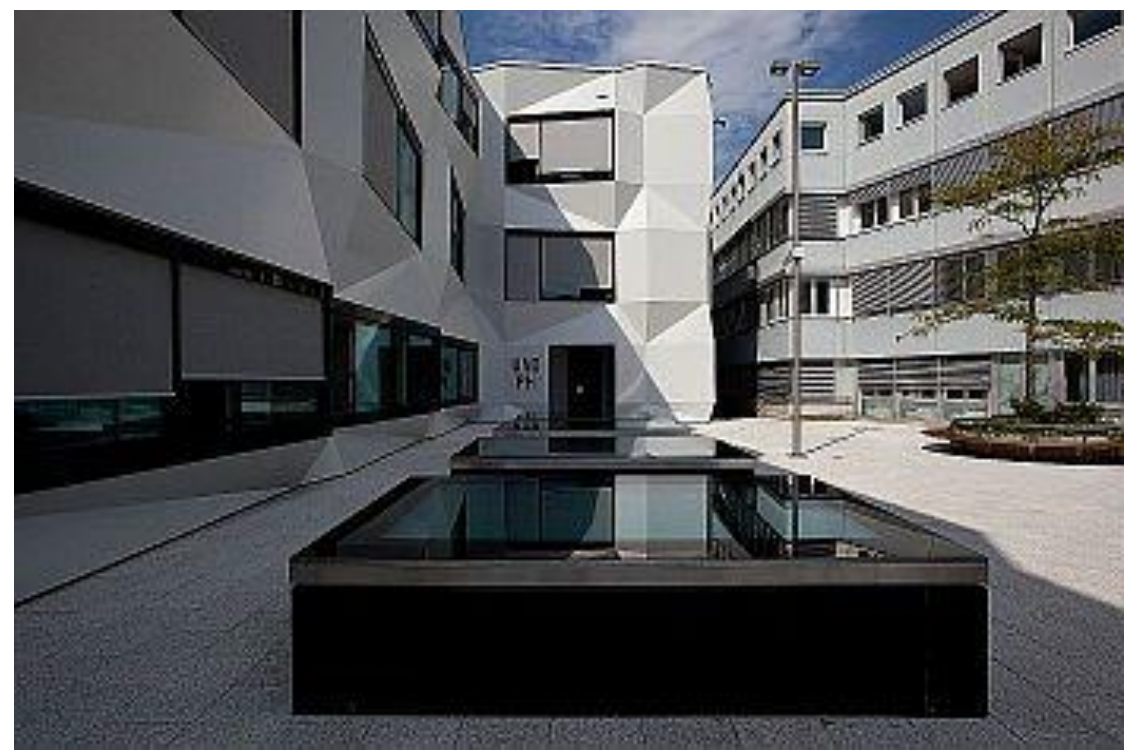

Abb. 11: Uni/PH Luzern : Der Innenhof des ehemaligen Postgebäudes (Foto: Niederer, ZHB Luzern)

Die Architekten nutzten geschickt die vorhandene innere Organisation des Gebäudes ohne größere Umbauten. Die stark frequentierten, öffentlich zugänglichen Bereiche wie Mensa, Lounge und Hörsäle befinden sich im Erdgeschoss bzw. im 1.Untergeschoss. Die Bibliothek nimmt das gesamte 1.Obergeschoss ein, darüber liegen die Büros und Seminarräume der Universität und der Pädagogischen Hochschule. Die Bibliothek umfasst 5.500 qm mit ca. 200.000 Bänden Freihandbestand (davon 30.000 Bände Studienbibliothek der PH Luzern, Zielbestand sind insgesamt 300.000 Bände) sowie 670 Arbeitsplätze in verschiedenen Bereichen der Bibliothek. Entlang der Fensterfronten nach Außen und zu den Innenhöfen befinden sich gereiht die Arbeitsplätze, es gibt dazu einen Doktorandenlesesaal sowie Gruppenarbeitsbereiche und -räume sowie einen Lounge-ähnlichen Bereich für die Kommunikation. Die zwei Lichthöfe bieten der Bibliothek Licht und Transparenz zu den öffentlichen Bereichen im Erdgeschoss. Die farbliche Gestaltung der strukturierten Wände erlaubt einerseits Orientierung im Gebäude, lässt die Räume aber auch wertvoll erscheinen, z.B. durch die Silber gestrichenen Decken. Die Architekten überzeugten den Bauherrn, die schlichte Lochfassade im Umfeld von Bahnhof und Kultur- und Kongresszentrum durch eine markante Außenfassade zu ersetzen, die durch ihre Faltung jeweils unterschiedliche Ausblicke zulässt und dem Platz vor dem Gebäude eine neue Bedeutung zukommen lässt.

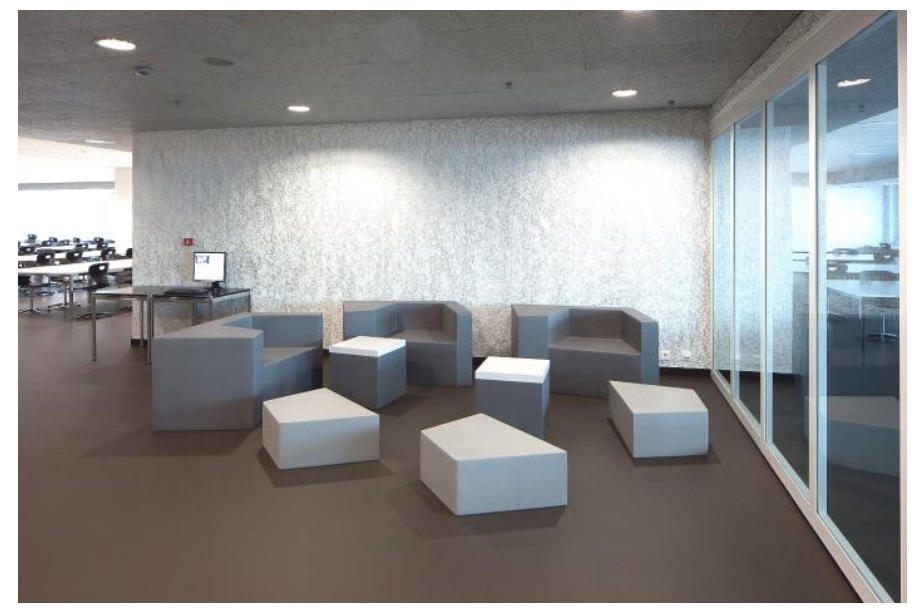

Abb. 12: ZHB Luzern : Leseecke im ehemaligen Postgebäude (Foto: Niederer, ZHB Luzern) 


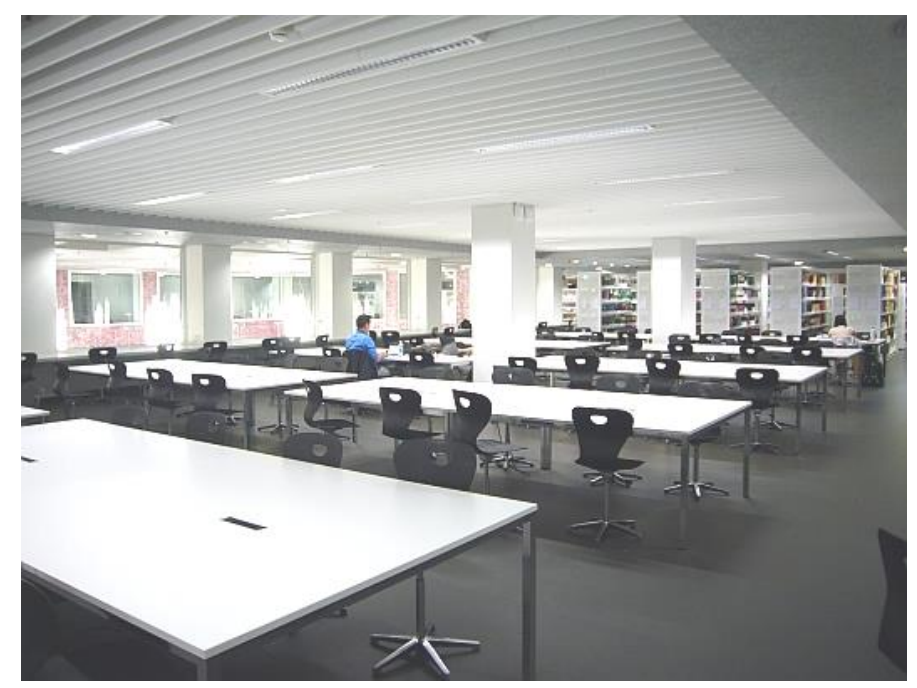

Abb.13: ZHB Luzern : Arbeitsplätze im Lesesaal (Foto: Feldsien-Sudhaus)

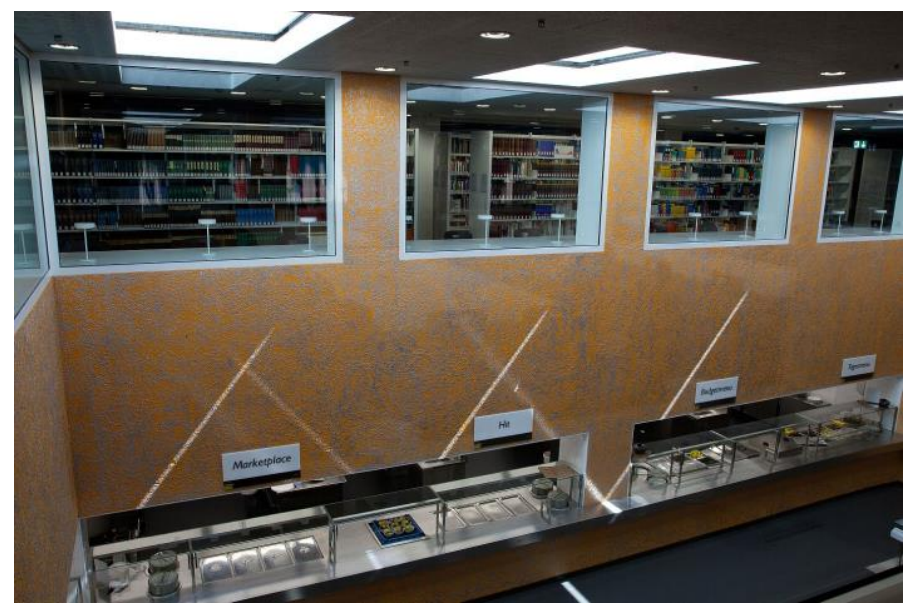

Abb. 14: ZHB Luzern : Blick in die Mensa zur Essensausgabe im EG (Foto: Niederer, ZHB Luzern)

\section{Kaisa House - vom Kaufhaus zur Universitätsbibliothek (Helsinki, FIN)}

In der Innenstadt von Helsinki konnte im Dezember 2006 die Universität ein ehemaliges Kaufhaus erwerben, um dort vier Fakultätsbibliotheken sowie die Undergraduate Library zu einer zentralen Universitätsbibliothek zusammen zu legen. Dabei wurden die Obergeschosse des Kaufhauses abgerissen und auf die erhalten gebliebenen vier Untergeschosse, von denen es auch einen Zugang zur U-Bahn-Station gibt, wurde die neue Universitätsbibliothek ${ }^{25}$ mit sieben Obergeschossen gebaut. Das Architektenbüro Anttinen Oiva Arkkitehdit Oy gewann 2008 den Wettbewerb. Das Budget belief sich auf 35 Mio. $€^{26}$ Die Architekten besuchten mehrfach das Vorgängergebäude mit seinem Parkhaus und entschieden sich, durch elipsenförmige Einschnitte in das Dach, in die Fußböden und in die Fassade Licht in die Gebäudetiefe zu bringen. Dies bietet gleichzeitig schöne Ausblicke auf

\footnotetext{
${ }^{25}$ Experience the New Helsinki University Main Library. Video 05.10.2012. URL: http://youtu.be/pTu4FTjILK8 [Stand 31.12.2012]

${ }^{26}$ Nykopp, Nicola: New Service Concepts and Library Buildings at the University of Helsinki. 2008. Vortrag 09.04.2008, 14. Seminar of LIBER Architecture Group LAG. URL: http://147.88.230.242/liberlag/PP_LAG_08/Wednesday/nykopp080408-def2.pdf [Stand 28.12.2012]
} 
das Zentrum von Helsinki. Dabei nimmt die Fassade ${ }^{27}$ die Gestaltung der umliegenden, gemauerten Fassaden auf.

Es wurden für die Bibliotheksnutzung 16.000 qm sowie für Läden und Geschäfte 5.600 qm geschaffen. Ein wichtiges Ziel für die Bibliotheksflächen war Flexibilität, um der Universität Entwicklungschancen an diesem Standort für die nächsten 50 Jahre zu sichern, zumal der innerstädtische Bauplatz sehr teuer ist. Möglicherweise zu teuer zum einfachen Lagern von gedruckten Büchern in der Zukunft? Aktuell verzeichnet die Bibliothek noch 500.000 Erstausleihen, so dass $30 \mathrm{~km}$ Regalböden für 1,5 Mio. Bände vorgesehen wurden. Zum Konzept gehört auch, für die verschiedenen Kundengruppen angemessenen Arbeitsraum zu schaffen, so z.B. im 7.Obergeschoss einen „Wohnraum“ für Wissenschaftlerinnen und Wissenschaftler, im 2.Obergeschoss ein Literatur-Café oder Raum für Gruppenarbeit. Eine repräsentative runde Treppe verbindet die Geschosse. Da die Geschosshöhe nicht sehr hoch ist, legten die Architekten die Treppe bewusst zwischen die Lichthöfe, um dadurch punktuell einen großzügigen Eindruck zu vermitteln. Am 3. September 2012 wurde das Gebäude eröffnet.

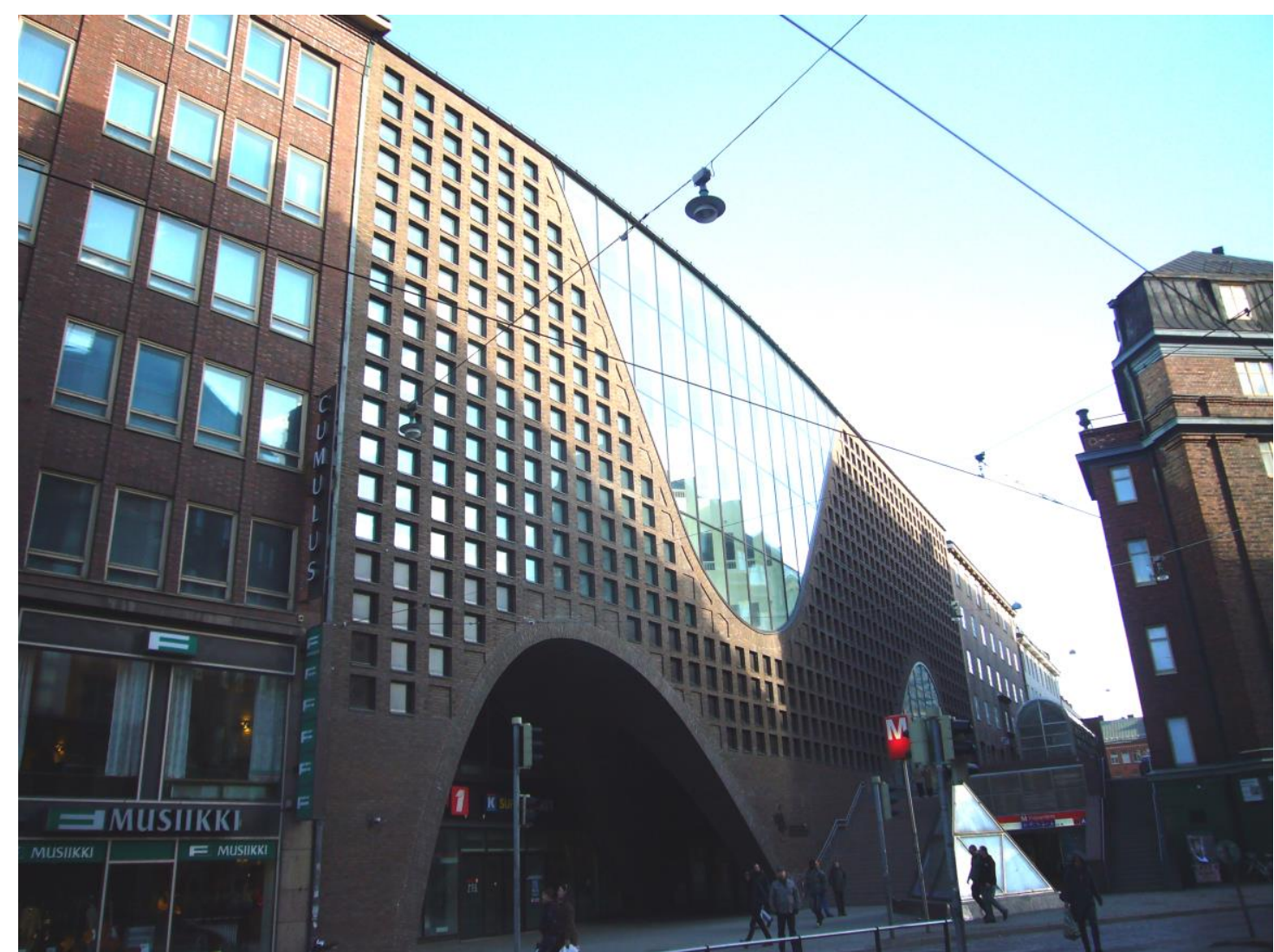

Abb. 15: Kaisa House Helsinki : Zugang zu den Shops, der Metro, dem Cafe von der Strasse Kaisaniemenkatu (Foto: Feldsien-Sudhaus)

\footnotetext{
${ }^{27}$ Kaisa-talo | Kajsahuset | Kaisa House. URL: http://www.helsinki.fi/kirjastot/kaisa_taloesite.pdf [Stand 31.12.2012]
} 


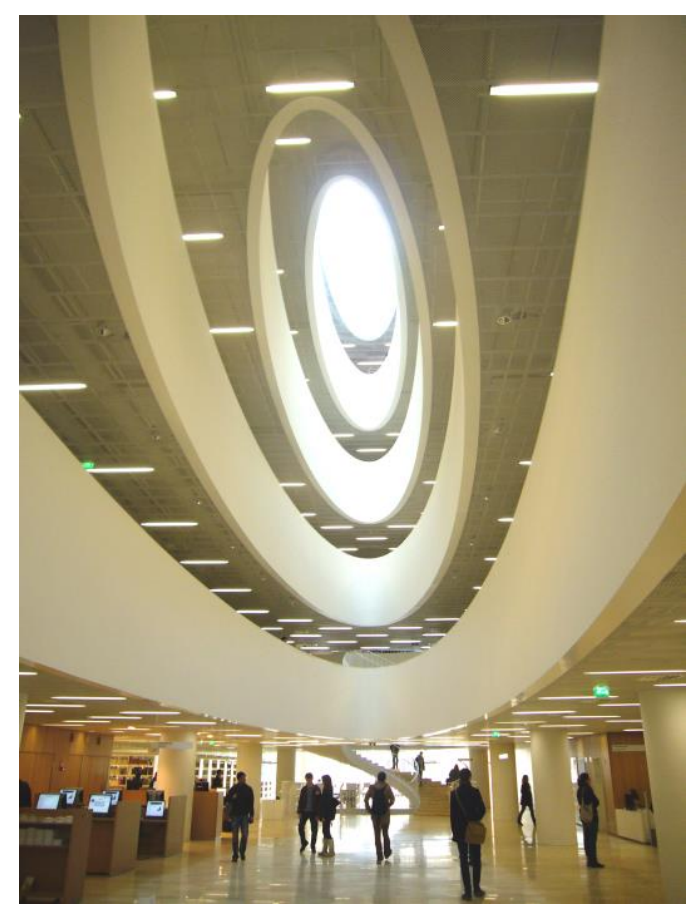

Abb. 16: Kaisa House Helsinki : Eingangshalle auf der Ebene der Strasse Fabianinkatu 30 (Foto: Feldsien-Sudhaus)

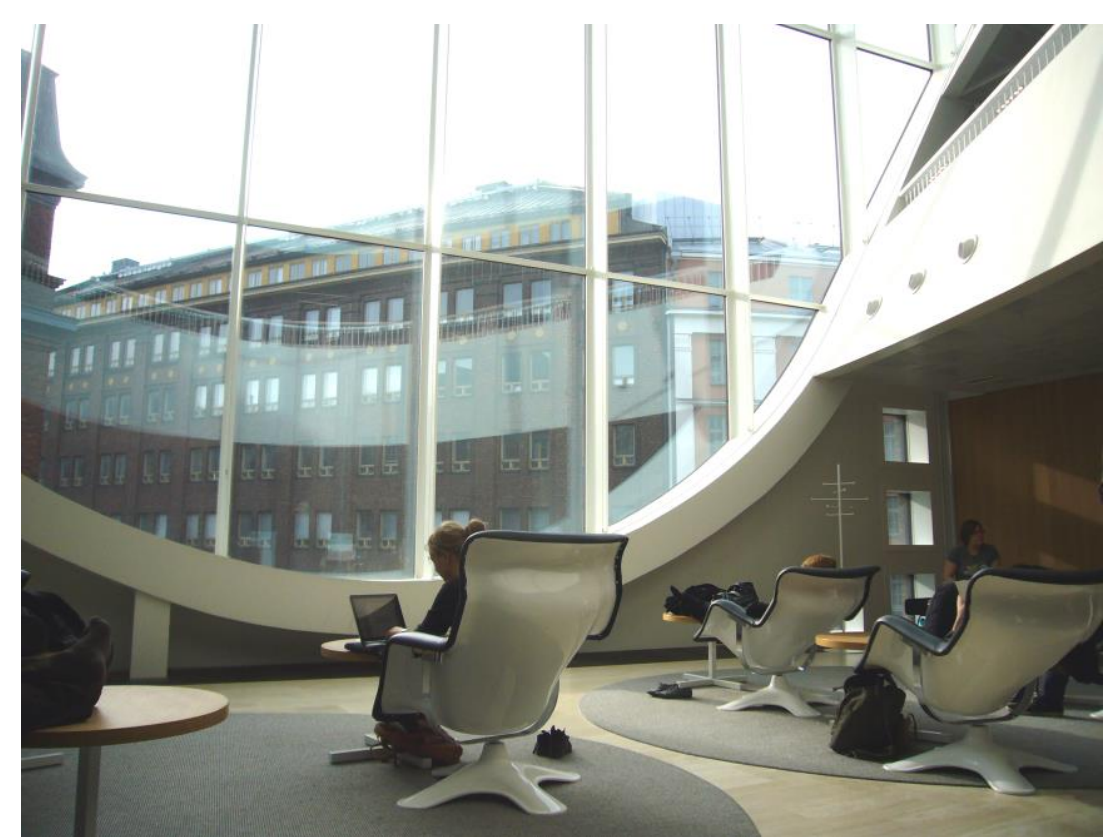

Abb. 17: Kaisa House Helsinki : Arbeiten und Relaxen mit Blick auf die belebte Strasse Kaisaniemenkatu (Foto: Feldsien-Sudhaus) 


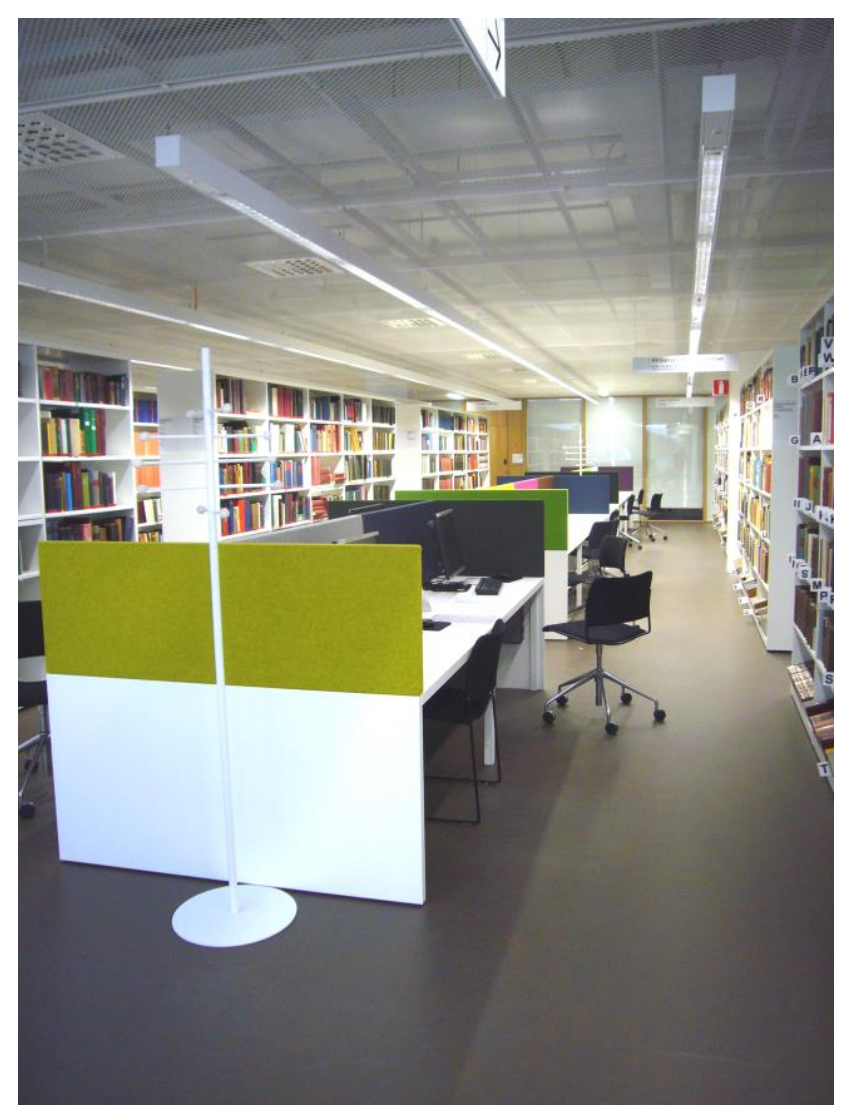

Abb. 18: Kaisa House Helsinki : Arbeitsplätze zwischen den Regalen (Foto: Feldsien-Sudhaus)

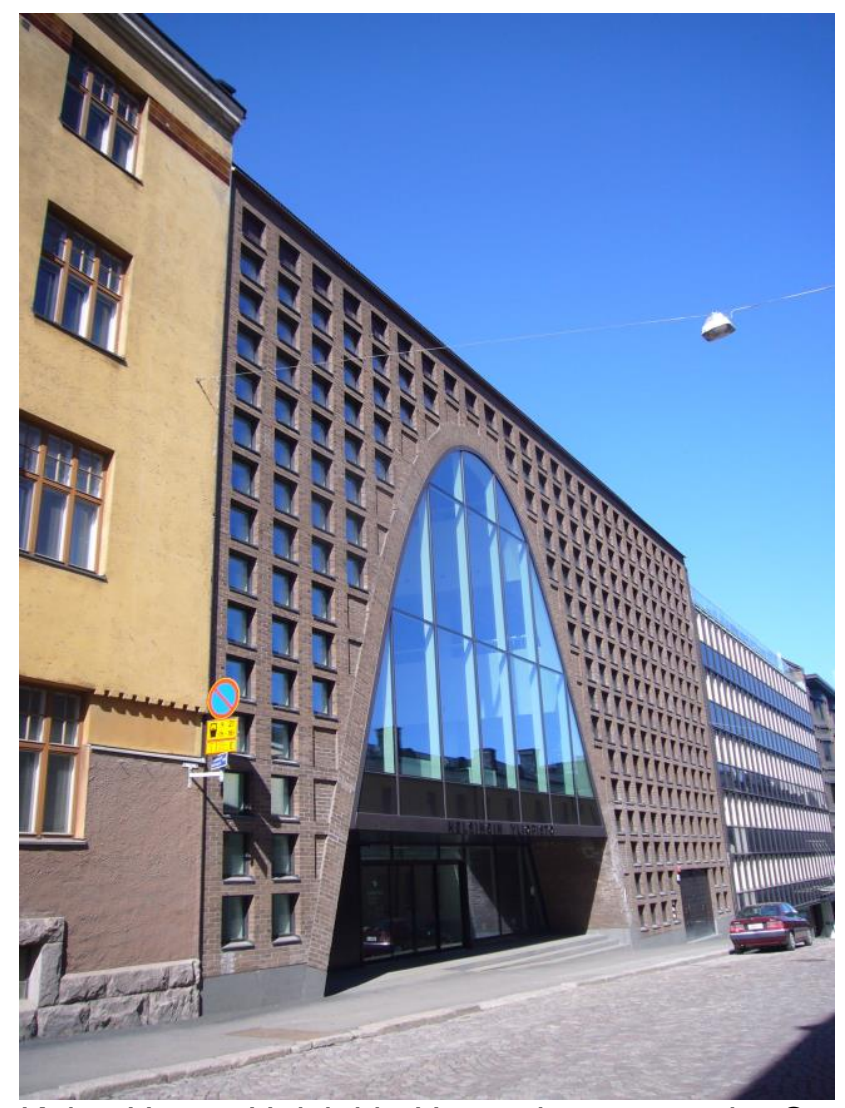

Kaisa House Helsinki : Haupteingang an der Strasse Fabianinkatu 30 (Foto: Feldsien-Sudhaus) 


\section{Dynamik der Entwicklung}

Die Beispiele zeigen deutlich, dass die technologische Entwicklung und die dadurch geänderten Kundenwünsche im Bibliothekswesen zu notwendigen Anpassungen der Gebäude führen. Dies gilt nicht nur für Gebäude, die älter als 30 Jahre sind, sondern auch schon für Bauwerke aus den 1990er Jahren. Wenn das vorhandene Tragsystem Entwicklungschancen bietet, kann eine veränderte Raumorganisation auf vorhandener Fläche ein Lösungsansatz sein. Dazu gehören der Ersatz der (sicherheits-)technischen Infrastruktur, grüne Technologien der Gebäudetechnik, nachhaltige Materialien sowie der Ausbau der IT-Technik. Flexibles Mobiliar ermöglicht es Bibliotheken, ohne großen Aufwand auf zukünftige Nutzungsveränderungen reagieren zu können.

\section{Literatur:}

Advisory Board Company. Redefining the Academic Library: Managing the Migration to Digital Information Services. Washington, D.C.: The Advisory Board Company, 2011.

Aymonin, David; Bisbrouck, Marie-Françoise; Joye, Nicolas; Rittmeyer, Mirjana: Learning center - the Lausanne example. Vortrag 24.03.2006, 13. Seminar der LIBER Architecture Group LAG. URL: http://147.88.230.242/liber-

lag/PP_LAG_06/Friday/AymoninRittmBisbrouck-EPFL.pdf [Stand 28.12.2012]

David Milling Architects: Clemson University : Cooper Library Masterplan. Camden, South Carolina, 2008. URL:

http://media.clemson.edu/library/lib_overview/plans/CooperLibraryMasterplan10312008.pdf [Stand 10.12.2012]

Gabler, Christiane: Vertriebszentrum wird Hochschule. In: Bauwelt , 103.2012; Heft 33 Erscheinungsdatum: 24.08.2012, S. 15-23

LAUC Committee on Professional Governance: The NextGen LAUC Member : A Report. 2011. Librarian Association of the University of California (LAUC)

URL: http://www.ucop.edu/lauc/committees/pg/cpg-report-2011.pdf [Stand 29.11.2012]

Redecke, Sebastian: Campus mit Teppichlandschaft. In: Bauwelt, 101.2010, Heft 13, S. 1423

Tamminen, Tuomo: On the student's terms. In: Helsinki University Bulletin HUB 3/12, S. 5257

Verfasserin:

Inken Feldsien-Sudhaus

Direktorin der Universitätsbibliothek

Technische Universität Hamburg-Harburg

Denickestr. 22

21073 Hamburg

+49 40 42878-3004/3204 Sekr.

feldsien-sudhaus@tuhh.de 